\title{
TOWARDS AN AUTOMATED POLISHING SYSTEM - CAPTURING MANUAL POLISHING OPERATIONS
}

\author{
Eugene Kalt ${ }^{1}$, Radmehr Monfared ${ }^{2}$, Michael Jackson ${ }^{3}$ \\ ${ }^{1}$ Wolfson School of Mechanical, Electrical and Manufacturing Engineering, Loughborough University, UK \\ ${ }^{2}$ Wolfson School of Mechanical, Electrical and Manufacturing Engineering, Loughborough University, UK \\ ${ }^{3}$ EPSRC Centre for Innovative Manufacturing in Intelligent Automation, Loughborough University, UK
}

\begin{abstract}
Advancements in robotic and automation industries have influenced many manual manufacturing operations. With a great level of success, robots have taken over from man in many processes such as part manufacturing, transfer and assembly. However, in other traditionally manual operations such as polishing, automation has only partially been successful, typically limited to parts with simple geometry and low accuracy. Automated polishing systems using robots have been attempted already by a number of industrial and research groups; however, there are few examples of deploying such a system as a part of a routine production process in high-technology industries, such as aerospace. This is due to limitations in flexibility, speed of operation, and inspection processes, when compared with manual polishing processes. The need for automated polishing processes is discussed in this article and the problem with the existing system was explained to be a lack of understanding and the disconnect from manual operations. In collaboration with industrial partners, a mechatronic based data capturing device was developed to accurately capture and analyze operational variables such as force, torque, vibration, polishing pattern, and feed rates. Also reported in this article is a set of experiments carried out to identify the polishing parameters that a manual operator controls through tactile and visual sensing. The captured data is interpreted to the operators' preferences and polishing methods and should then be included in the design of an automated polishing system. The research results reported in this article are fed back to an ongoing research project on developing an integrated robotic polishing system.
\end{abstract}

Keywords: Capturing Manual Operation, Automated Robotic Polishing, Force and Motion Capture

\section{INTRODUCTION}

In the manufacturing industry, mechanical finishing plays a vital role in the development of product surface quality and final geometry [1]. Mechanical finishing typically includes deburring, grinding, polishing, buffing, and final visual inspection of a workpiece. These processes are generally performed at the final stage of the manufacturing process of a component or product and may represent up to a third of production time in some industry [2].

One of the main reasons for polishing is to improve surface finish by removing minimal amounts of material and to smooth a particular surface until obtaining the desired surface finish (i.e. roughness or aesthetic aspect) without affecting the geometry of the workpiece [1-3].

Mechanical polishing, as common finishing methods, refers to the removal of fine layer of material by means of abrasive tools to reduce the surface roughness to the desire level (e.g. roughness average or $\mathrm{Ra}$ ). Smoothing of surfaces generally involves removing scratches, machining marks, pits, and other defects or features to obtain a uniform surface roughness evenly distributed throughout the part surface [3].

The polishing process is very important and highly used in aeronautical industry, whether it is to meet mechanical properties and design specifications, such as friction, or to meet the desired visual aspect. For example, a hydraulic turbine produces electricity by turning energy from water into kinetic energy for an electric generator [4]. The main factor that affects the efficiency of these hydraulic turbines is the friction between the water and turbine blades. The level of fiction will depend on the quality of the surface finish. Thus, improving the surface roughness of a turbine blade will significantly decrease the friction and increase the efficiency of the turbine. In addition, by meeting design specifications and surface quality, polishing processes can improve the service life of a component. Therefore, additional processes such as polishing are required after initial machining processes [5].

Despite the growth of automated technologies used in modern industry, polishing processes are still mainly carried out manually. Manual polishing typically involves a highly skilled worker holding a workpiece or a polishing tool in order to: remove a layer of material; remove scratches or machining marks; shape curves or radii; or deburr and break sharp edges. Skilled human operators have the advantages of adapting quickly to changes, to be flexible, and ability to learn from their mistakes. However, it can take many months to train a new operator. In addition, the working environment is unhealthy for the operators, due to exposure to dust, vibration and noise. In many cases, lengthy manual polishing processes lead to "vibration white finger" or other musculoskeletal diseases (MSD). Current regulations stipulate that operators must wear safety glasses, respiratory-protective equipment and take regular breaks. 
Moreover, some companies may have difficulty in recruiting and training sufficient numbers of highly skilled manual workers to meet their requirements [2,6-10].

These are some of the reasons why some industries are strongly motivated to seek and implement alternative solution in their manufacturing processes to improve the working environment, meet targets, and drive costs down, whilst keeping the same level of quality or better [2,8-10].

Many industries already benefit from the advances in multiaxis machining to produce parts to precise tolerances and specifications instead of polishing process $[9,10]$. Industrial robots have also been widely used to perform precise, repetitive tasks in carefully controlled environments, but robots performing a fully autonomous polishing operation may not be cost-effective [11]

Some automated solutions have already been proposed to assist or replace human operator. However, these solutions typically lack flexibility and dexterity that are provided by human operators. For example, some of the polishing skills that are particularly challenging to automate include rapid reasoning and decision making based on visual inspections, and fast adjustment of the polishing patterns, e.g. when a surface defect is identified.

To develop a robust automated polishing system, it is essential to incorporate human skills into the automated systems. Therefore, it is necessary to understand and capture these manual skills to be able to build the automated system on that basis.

Following a review on the current automated polishing system, it was found that the manual skills have largely been neglected when designing such automated systems. To the author's knowledge, no automated solutions has yet been developed to understand the human skill in this domain of industry and to implement them within an automated polishing system. This has contributed to the fact that currently there are not many robust automated polishing systems used in industries as part of their production systems.

Therefore, it was envisaged that the development and implementation of an automated polishing system should starts with a comprehensive understanding and assessment of the manual processes and operators' skills.

This article reports the first part of this research focused on capturing process parameters of manual polishing operations. In this article, the authors describe the design and development of a device to enable capture of manual polishing parameters and the polishing pattern used by skilled operators for complex parts. The captured parameters are then used and analyzed in development of an automated robotic polishing system as part of an ongoing research project, which is aimed to be published in a following article.

\section{RELATED WORK}

Mechanical polishing includes a wide range of technologies and processes, such as abrasive blasting, mass finishing, chemical-mechanical polishing and ultrasonic polishing. Three technologies (robot end-effectors, computer numerical control (CNC) and mass finishing) are mainly used in industry and are currently in development in research laboratories. Robotic arms are used for their flexibility and ability to imitate human motion. However, they suffer from low stiffness and high cost and complexity when integrating sensors $[12,13]$. CNC machines are known for their high precision but are high-cost and have no intelligence, flexibility, or adaptability $[10,14]$. Finally, mass finishing technologies are capable of polishing large batches of small parts, but suffer from a long machining time (i.e. 6 to 8 hours), and low accuracy, and still require an experienced operator $[15,16]$.

Extensive work has already been carried out on various technologies used in modern industry and ongoing research (from basic concept to design of a new automated system). For example, Dickman [1] details the basic concepts and approaches for surface finishing and preparation. Moreover, Keyton [9] developed a classification of usage of mechanical finishing systems in industry and speculates on the future of automated mechanical polishing. Finally, Murphy [17] has published a general review of different work published in surface finishing. These include polishing, buffing, blasting, cleaning, coating, and plating.

Before automating such processes, Tsai [18] and Besari [3] advised to carry out an exact and clear measurement of the polishing parameters. This is because, the successful design and implementation of an automated polishing system using the correct level of automation is required through the study of the polishing process. In the following paragraph, a few examples of work carrying out automated polishing are presented.

Axinte [10] and Guiot [14] have worked with CNC milling machines to carry out polishing operations. CNC milling machines has the advantage to produce high-quality components, but at high cost with no real-time adaptation.

Tsai [18] developed a software solution for automated polishing system which adjust the path depending on the geometry of the workpiece and the grain size of the tool.

Researchers in the Singapore Institute of Manufacturing Technology (SIMTech) and Nanyang University have worked on an automated system for grinding and polishing operations for the refurbishment process of turbine blades [8,19-25]. The authors developed two solutions, SMART Robotic System and a self-compensated closed loop RealTime Robotic Polishing System (RT-RPS). Their systems integrate an in-situ measurement system for measuring the true profile of each workpiece, which then match to a template to generate new path and machining parameters, before starting the grinding and polishing process. 
Their approach of measuring each workpiece before each operation and matching them to templates to create a new set of parameters may not be appropriate - as this solution is yet to be proved effective. Also, similar to many research trends, a major focus is placed on the technical aspect of automated polishing with insufficient investigations on the influence of manual processes.

Recently, Du [27] developed a compliant end-effector and a method for force-position control for automated polishing operation of titanium alloy samples. Their system uses a tool path generated with CAM software (i.e. the authors used a CNC g-code program for the robotic arm). The force is captured and transformed into a vector to calculate the polishing force. Then a position vector is calculated based on the force data and the current position of the end-effector. Finally, this information is sent to the robot controller to correct or adjust the position and orientation of the endeffector. It was also interesting to notice that a study of the manual operation carried out, was limited to the force applied on the part by an operator. This was to justify to define the amount of force required [27].

In the commercial sectors, businesses have already introduced and proposed automated systems such as CNC grinding or mass finishing. However, the implementation and usage of robotic polishing systems is still limited due to the complexity of the process. Nonetheless, some solutions for robotic polishing are available for industries [28-31]. These solutions were designed to reduce the polishing time and cost while removing human operators from the process to achieve the same or better quality of surface finish.

With the use of CAD/CAM software and in-situ metrology, automated polishing has advanced significantly in the last few years. However, there are still major barriers in deployment of these technologies which result in wide usage of manual processes for individual parts. The polishing time is still long in most industries. Therefore, the cost associated with manual or automated processes is also high. There is also insufficient adaptability and intelligence in the existing automated polishing systems which prohibits the development of industrial scale systems; particularly when compared to skilled operators who can work faster, are more adaptable and flexible, and can achieve the same or a higher quality of surface finish.

Alternative semi-automated solutions are also available through applications of manually handled fixtures [32] or haptic devices [33] controlled by a skilled operator. While these solutions can reduce the risk of injury or the training time, they may not be cost effective and they still rely heavily on operator skill and experience.

The review of the current state of the art indicates that, despite the existence of a number of automated solutions, industries are still heavily relying on manual process as faster, more flexible, and more cost-effective approach to achieve the desired surface finish.

\section{UNDERSTANDING MANUAL POLISHING PROCESSES}

This research initiated with collaboration with a polishing SME company, specializing in high precision aerospace components. The polishing process specifications and the sequence of operations used in this research has been captured through numerous company visits, interviews, and video analysis of manual processes occurred in the partners' site. The research work started by capturing of high level processes to understand the dependency of the business on manual polishing operations. The research continued by capturing of low level polishing parameters and the influence of operators' experiences on standard machining processes.

In a standard operating procedure for small complex metallic components, an operator holds a workpiece (typically by hand) and pushes it against an abrasive belt or a wheel to remove defects or improve the surface texture. The quality of the process is monitored visually during and after each process. The operator adjusts machining parameters accordingly in real-time through visual inspection and tactile feedback.

The complete process sequence of one component would typically be carried out by a single skilled operator, starting from a high abrasive grit (i.e. grinding) and ending to a low abrasive grit (i.e. polishing, buffing). Each operator is highly skilled and can work on multiple component or processes, depending on the production needs. The operator can locate superficial defect on the surface and adapt parameters in real-time, to provide a high quality of surface finish in minimum amount of time. Due to human errors, some parts may have to be reworked several time until the quality of surface finish is satisfactory. Operators could be exposed to health and safety risk such as white finger disease due to intense vibration and forces involved in these processes.

\section{CAPTURING MANUAL POLISHING OPERATIONS}

Manual polishing processes are involved a great number of parameters and variables related to the workpiece, polishing tool, and the operators, as illustrated by Fig 1.

Some of the variables include the contact force between the part and abrasive tool used to remove defects or layer of material; the motion speed of each polishing action; the machining path or operator movements; and the control of the quality and geometry of the part. Other variables such as vibrations, heat, noise, abrasive wear, and hand grasp that are beyond the scope of this article, also provide important feedback to the operator to adjust the process parameters. 


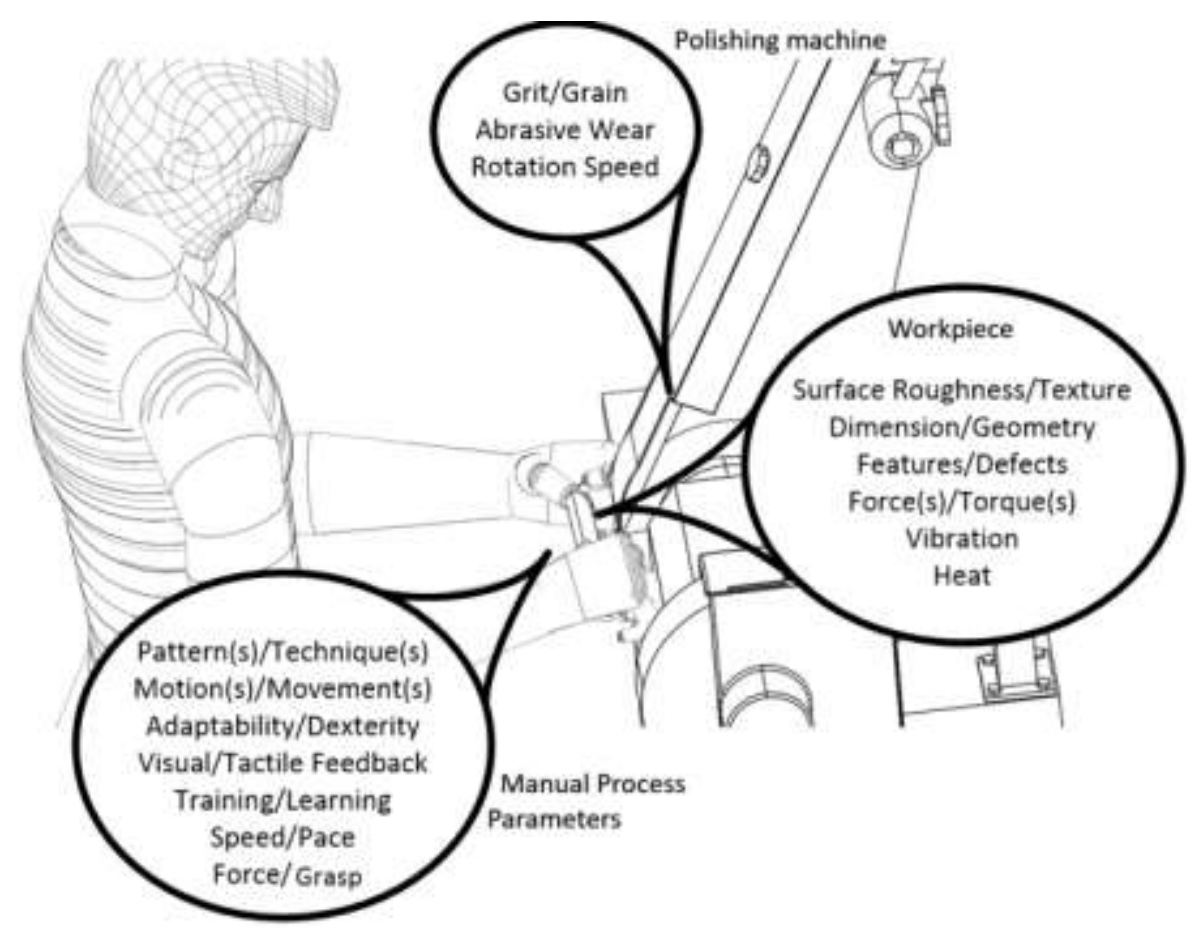

Fig -1: Example of Manual Polishing Parameters and Variables

In addition to the machining parameters and operation variables, a number of "unwritten polishing methods" in form of patterns and polishing techniques were identified that are used commonly by the operators. For instance, changing force or feed rate when removing defects from a known position on the workpiece.

In order to develop an automated polishing system, it was important to understand how and under what circumstances the operators change the polishing parameters and their polishing patterns. For this purpose, a set of experiments were designed to replicate the industrial polishing processes at laboratory environment. A device (as a fixture) was designed and developed to be used by skilled operators to facilitate the data capture. A number of sensors were embedded into the device to enable monitoring the machining parameters and the process variables during the polishing operations, as illustrated in Fig 2.

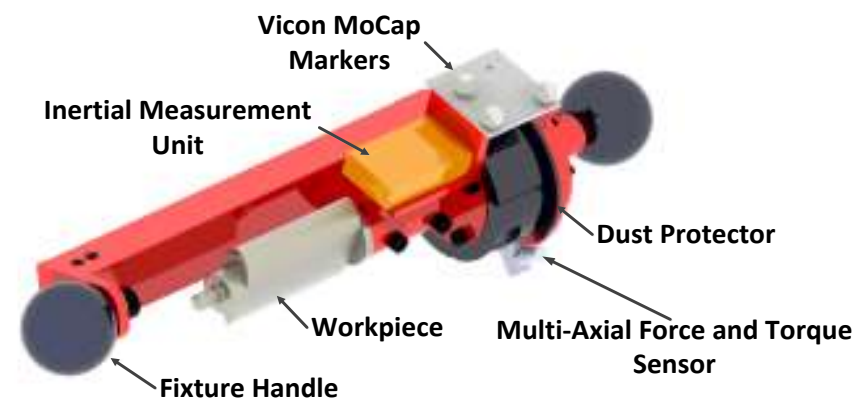

Fig-2: Design of the fixture to capture manual polishing parameters

Forces and torques applied during the polishing operation were captured using a multi-axial FTS (force torque sensor Shunk Gamma [6]). The sensor was able to generate three forces $(\mathrm{Fx}, \mathrm{Fy}, \mathrm{Fz})$ and three torques $(\mathrm{Tx}, \mathrm{Ty}, \mathrm{Tz})$ in realtime.
The part movements and the polishing pattern were captured using a Vicon motion capture system [7]. Reflective markers were placed on the fixture to be tracked by two cameras placed around the experiment environment. The captured pattern provided information about the operator's hand motions and then helped defining the trajectory of potential automated robotic polishing system.

The acceleration and the orientation of the part movement were captured using an inertial measurement unit (IMU XsensMTw [8]), which monitored the orientation of the fixture through gyroscope and magnetometer. The vibrations generated during contact with the abrasive belt, and the speed used by the operators were also captured through the accelerometer in this unit (not reported in this article).

\subsection{Calibration Process}

There are fundamental differences between standard manual polishing processes when operators hold a part by hand, or holding the fixture containing part and the sensors.

The fixture was designed ergonomically and made from very light material to reduce the potential errors due to gravity force. The skilled operators confirmed that after a number of trial processes, they can comfortably use the fixture as part of their normal polishing process. However, to replicate the polishing process at a control environment as close to the actual process as possible, a set of calibration process was implemented to interpret and adjust the output data. The calibration process facilitates accommodating the errors introduced by the weight of the fixture, the unconventional part handling, and the change in the magnitude and the orientation of the axial forces applied by an operator. 
A set of systematic experiments were carried out to provide a reliable reference point for the various measurement sensors mounted on the fixture. These experiments provided a benchmark to interpret the sensors' data in the context of manual polishing operations. One set of experiments focused on the calibration of the multi-axial force and torque sensor, and the second set on benchmarking the data extracted from the motion sensors.

Using a laboratory set-up, the precision of the FTS for a single known force at different sampling rates was measured at between $0.02 \mathrm{~N}$ and $0.06 \mathrm{~N}$. This represented less than $5 \%$ error, which deemed to be acceptable for this research. Furthermore, the multi-axial forces and the resultant torques were also measured when a constant static force was applied to the part at known direction, while the fixture was rotating in a measured speed using a robot arm, as shown in Fig 3. The force distributed along Fx, Fy, and Fz differently but the resultant force remained the same at any point on the surface. However, the value of the torques were linked to the distance between the load contact point and the FTS. By measuring the distance, the values of the resultant forces and torques were offset through calculations.

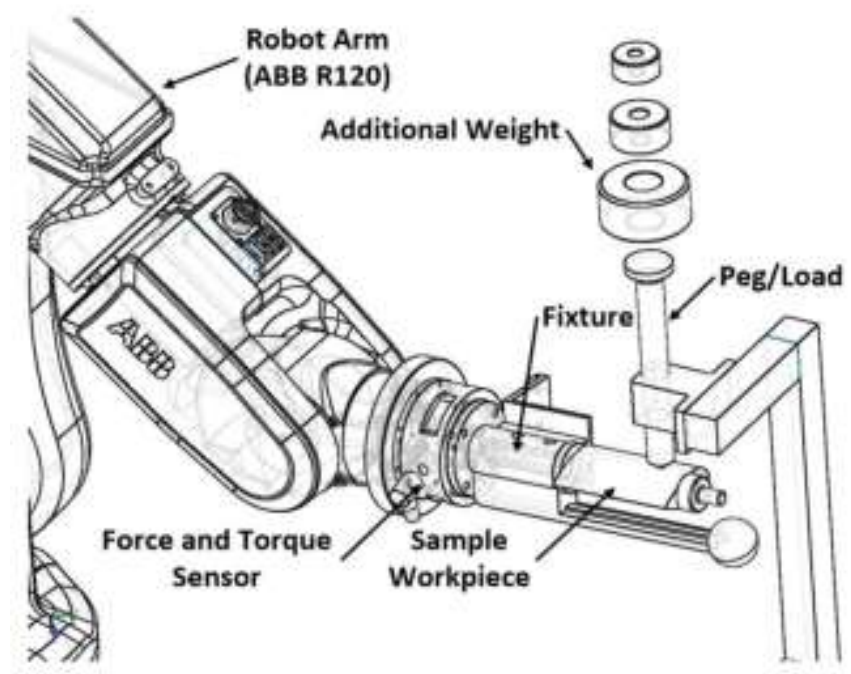

Fig-3: Calibrating the fixture for multi-axial force and torque

The same approach was implemented to calibrate the IMU and the 3D motion capture sensors. The direct data collected from the motion capture system defined the co-ordination of the fixture's reference point in a 3D space, as illustrated by Fig 4. Following an off-line analysis, this data was interpreted as the polishing pattern, the speed of the motion at a given coordination, and the time of the process.

\section{LABORATORY EXPERIMENTS}

To understand fully the polishing parameters, process variables, and operators' preferences in a manual polishing process, a number of experiments were designed at laboratory level. Running experiments at a controlled environment (in oppose to the industrial site) enabled: a) minimizing the impact of other events carried out at the site, b) repeating multiple experiments at specific sequence necessary for our experiments. Therefore, skilled polishers were brought in to the laboratory to carry on the experiments.

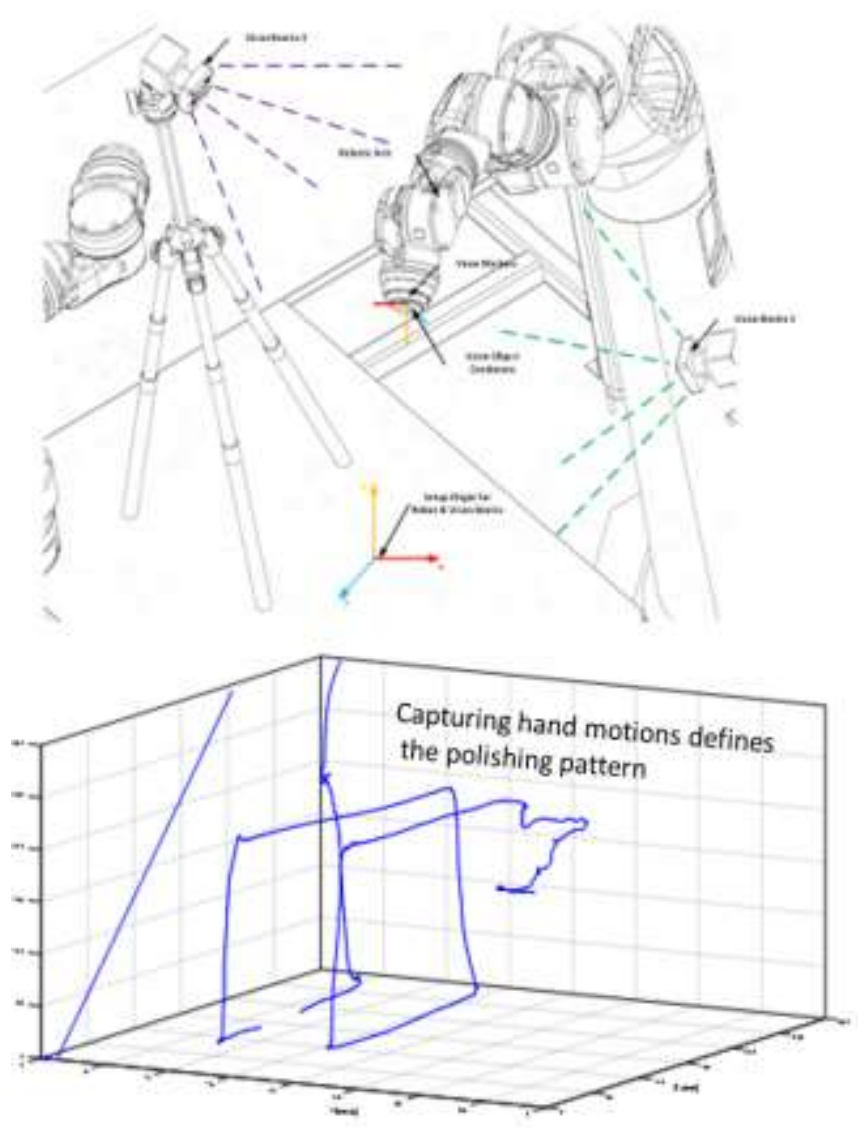

Fig-4: Calibrating for the pattern and speed of motions

The experiments were focused on complex small metallic components such as those used commonly in aerospace industry. Further focus was placed on two major polishing processes frequently performed in this sector of industry: a) removing a layer of material from surface to improve general surface finish, and b) removing surface defects.

Three sets of experiments were carried out by two highly skilled operators, including grinding and polishing operations on small metallic sample parts. The parts were designed to be similar to industrial components typically used at aerospace industry (actual parts were not used due to business sensitivity).

In experiment 1 , the techniques and parameters used by the operator for grinding operation were captured. The same tests were carried out at experiment 2 for polishing operations, aiming at removing heavy grinding marks and improving the surface finish. Key differences between the two experiments were expected in respect to the machining parameters and the motion patterns. In experiments 3 , the techniques used by the operator were captured to remove light surface defects at known locations on a part, while maintaining the surface finish and texture. 
The experiment set-up comprised of a stainless steel part mounted onto the fixture and a dual-linisher machine (with an aluminum oxide $\mathrm{P} 240$ grit polishing belt) to polish the surface of the part, and two ViconMoCap cameras installed at each side of the linisher machine, as illustrated by Fig 5 .

During the first set of experiments, the operators removed a relatively thick layer of material from the surface of the part to eliminate the milling marks on the part's surface.

Grinding and deburring were the main processes carried out in this experiment. In the second sets of tests, operators had the task of removing heavy grinding marks and improving the surface aesthetic. A different grade polishing wheel was used to finish the surface texture.

Similar to the last experiment, it was observed that a different polishing pattern was used to deburr the trailing edges. In addition, the captured data indicate that the operator force and movement remained constant during the two experiments, however, the speed varied to adjust the feed rate and therefore material removal rate.

In the third experiment, surface defects were intentionally applied to the parts at known locations. Imitating the re- work process in real case scenarios, the operators were asked to remove the defects and then maintain the surface quality, similar to experiment 2.

Each experiment was repeated several times by each operators and the collected data were analyzed after experimentation. The data from each sensor were merged based on the process time, which was the single shared entity between all the sensors. Fig 6 illustrates a snapshot of the data captured from the experiments.

\subsection{Pattern Identification}

The results of the experiments indicate a number of techniques that manual operators typically use to maintain consistency and accuracy in their polishing processes.

The data output from the force and torque sensor and the MoCap cameras indicated three distinctly different techniques used by the operator, as illustrated by Fig 7. The first technique involved applying a static force against the abrasive wheel (Pattern A - constant pressure).

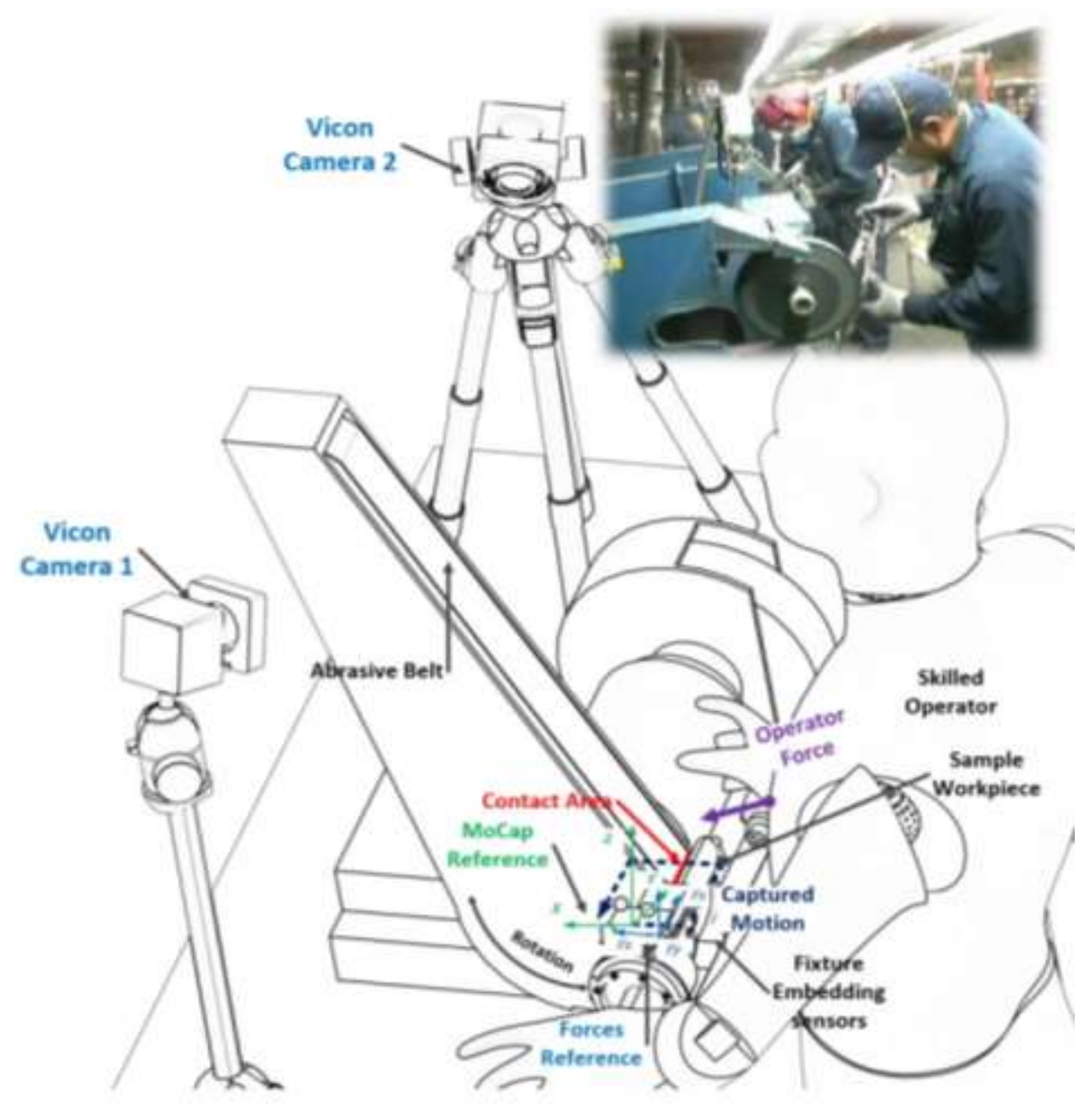

Fig-5: Laboratory test rig 

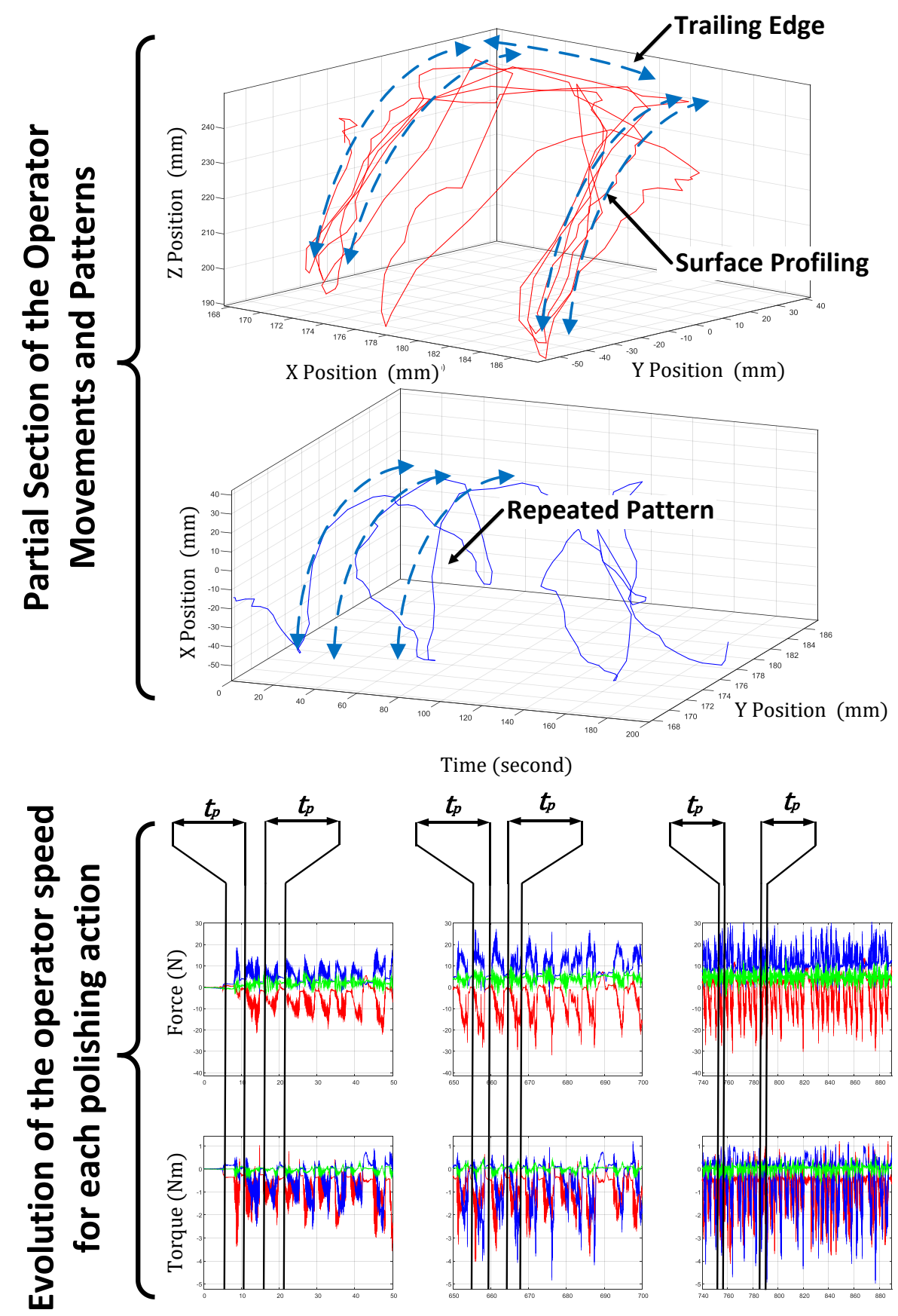

$t_{p}$ Duration 1 polishing action (sec.)

Fig-6:Captured operator technique and polishing parameters

The second identified technique showed a horizontal movement along the length of the part, while maintaining constant and perpendicular force applied to the abrasive tool (Pattern B - linear translation). In a line by line pattern, the complete surface of the part was covered, while no contact with the abrasive tool was made when moving from one line to another.

This technique is often used in industry to finish edges or remove studs. Results from the force and torque sensor indicates involvement of a combined force and torque (Fx, $\mathrm{Fz}, \mathrm{Ty})$. This is due to the perpendicular contact of part with the abrasive tool (Fx, Ty) and the associated horizontal movement (Fz). The MoCap cameras indicated a reciprocating motion that incrementally shifting to cover the part's surface. The quality of this technique is related directly to the operator skill level and experience.

The third technique indicated was the tracking across the surface's curved profile, along with the width of the part (Pattern C - surface profiling). In this method, operators continuously reposition the pivotal axis of the part in 2 dimensions to maintain a perpendicular contact point (or line) between the part and the abrasive tool. 


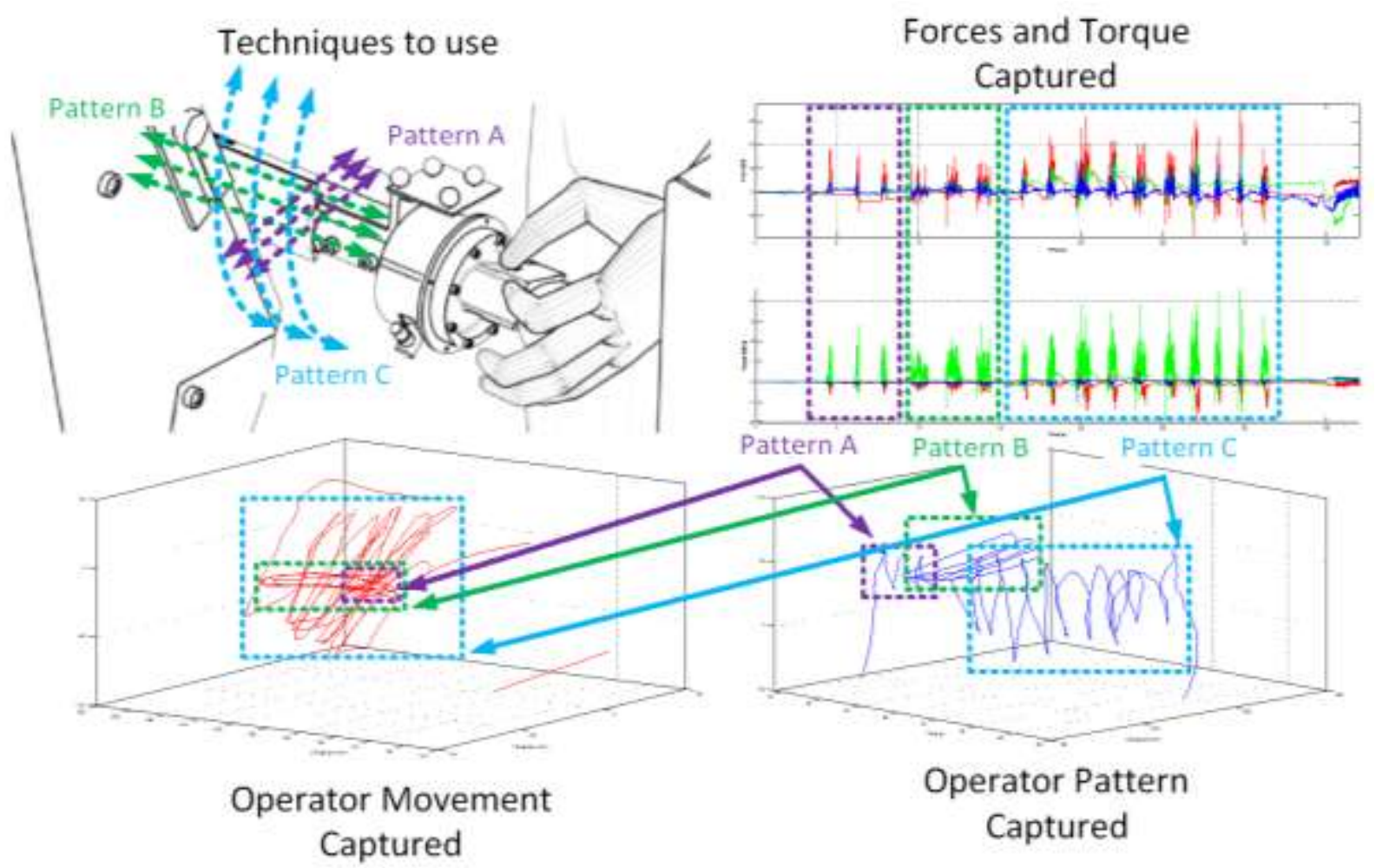

Fig 7:Motion patterns used by the skilled operators

The captured path by the MoCap cameras indicated resembled the CAD model of the part profile. The polishing path was parallel with incremental shifts occurring outside of the part edges. The sensors recorded three force values (Fx, Fy, Fz) and two torque values (Tx, Ty). This is due to the change of the workpiece orientation (Fx, Fy) and motion necessary for maintaining a perpendicular contact $(\mathrm{Fz}, \mathrm{Tx}$, Ty) with the abrasive tool. This pattern was found to be highly skilled operation that could cause additional surface defects on the parts due to human errors.

\section{TOWARDS A NEW AUTOMATED POLISHING SYSTEM}

Currently, there are a number of robotic polishing prototypes developed by various industrial and research groups. It was discussed that the main success of existing systems has been in the polishing of simple parts, and that currently no system is deployed at an industrial production level for more complicated components.

The current automated systems typically use a robotic arm that follows a trajectory based on parts' CAD model. In addition, a constant force feedback is received from the point of contact with an abrasive tool that enables adjusting the trajectory based on the force threshold predefined for the system. However, in the manufacturing of high-value components, the CAD file of a part is often not available to the polishing suppliers due to information sensitivity, the robotic polishing must therefore be pre-programmed based on individual components. This has proved to be impractical and time-consuming at an industrial level.
The findings from capturing manual polishing operations indicate a number critical design consideration during the development of automated polishing systems.

Three polishing patterns were identified in manual polishing operations (constant pressure, linear translation, and surface profiling). For polishing parts with complex surface curvatures, it was evident that skilled operators tend to follow the profile of the workpiece surface while keeping a constant force with perpendicular contact angle to the abrasive tool. A normal contact angle minimizes additional torques applied to the part and therefore makes the part handling easier and more stable. While skilled operators simply detect and rectify misalignment of the contact angle, maintaining a normal contact point could be a major design issue when polishing complex curvatures using a robotic arm. It was observed that the speed of data exchange could hinder the polishing performance. While humans can simply readjust the polishing motion in real-time, an automated system may fall behind when a complex surface is being polished. Therefore, it was concluded that an automated system requires an additional feedback loop to vary the process speed (through feed rate) in accordance with the system performance, to allow collecting and applying all sensor data in real-time.

It was observed that visual feedback is extensively used by manual polishers to assess the quality of the polished surface and locate potential defects between polishing passes.

Machine vision technology can be deployed as part of an automated polishing system to replicate the visual feedback in a similar way. However, the speed of such feedback is expected to be much lower than those generated by human. 
However, this could be compensated by the need for less rework due to the consistency of the automated processes.

Furthermore, it was observed that in addition to surface profiling, operators polish thin features of the parts, such as the trailing edges, using a linear movement (Pattern B). This technique allows the operator to deburr or straighten the edges, or to remove material or features that would not be removed with surface profiling (Pattern $\mathrm{C}$ ). It was observed that this approach is used due to the complexity of the geometry and the limitation of the operator's dexterity and his/her control over parameters. For instance, it was observed that polishing a thin/sharp edge would require extremely accurate motion and force control by the operator to avoid damaging the geometrical features of the part. To compensate the need for such high level of accuracy, operators change their polishing pattern (from Pattern $\mathrm{C}$ to B). However, it was envisaged that a robotic arm would be able to remove a layer of material on the whole surface in one routine, without the need for changing patterns. This is providing sufficient sensor feedback is received about the surface profile. Hence, a gradual declination of the machining speed should be embedded to the automation system when approaching thinner part of components. This should be based on the polishing forces being monitored continually and not the pre-existing data such as component CAD model, which was explained earlier that is often not available.

Analyzing the experiments' results, it was understood that polishing force (and its associated torques) is an important variable that operators tend to monitor and keep constant. The reason seems to be the fact that large magnitude of tactile sensing feedback generated by the force is easy to detect and control for a skilled operator. The torques generated during the polishing process is also tent to be maintained in one direction by adjusting the contact point (or line) with the abrasive tool. Fig 8 illustrates the control of the contact force in an automated system.
Therefore, to vary material removal rate, operators tend to maintain a constant polishing force and vary speed or feed rate. It was envisaged that a similar approach can be adopted for the automated system. For instance, during a rework process to remove surface defects, the material removal rate should be increased in the region of the defect. This could be achieved by increasing contact force or by reducing feed rate.

It was concluded that similar to the manual operation, the speed of the polishing process in an automated system could be better and more accurately controlled than the polishing force. In an automated robotic defect removal process, varying force would require integration of a vision system (to identify any defects' location) and a FTS (to measure force) to the robot. However, the varying feed rate would eliminate the need for the force control feedback.

Moreover, vibrations in polishing operation were found to be unproductive element that could cause tool wear and damage surface quality. However, vibration is also used by the operators through tactile feedback as an indication for the magnitude of polishing force and feed rate. In an automated robotic polishing system, the stiffness of robot arms would negate the vibration issue [29,30]. However, additional sensors would be required to replicate the tactile feedback that operators receive from vibrations. Replicating vibration feedback in beyond the current scope of this research.

In addition to the tactile feedback, skilled operators also use visual and auditory feedback to monitor and adjust polishing parameters in real-time. Auditory feedback could be used by skilled operators to identify tool wear, excessive machining parameters, and change in material. However, auditory feedback can be difficult to use consistently due to the mandatory use of personal protective equipment (PPE). Therefore, the auditory feedback is excluded from this research.

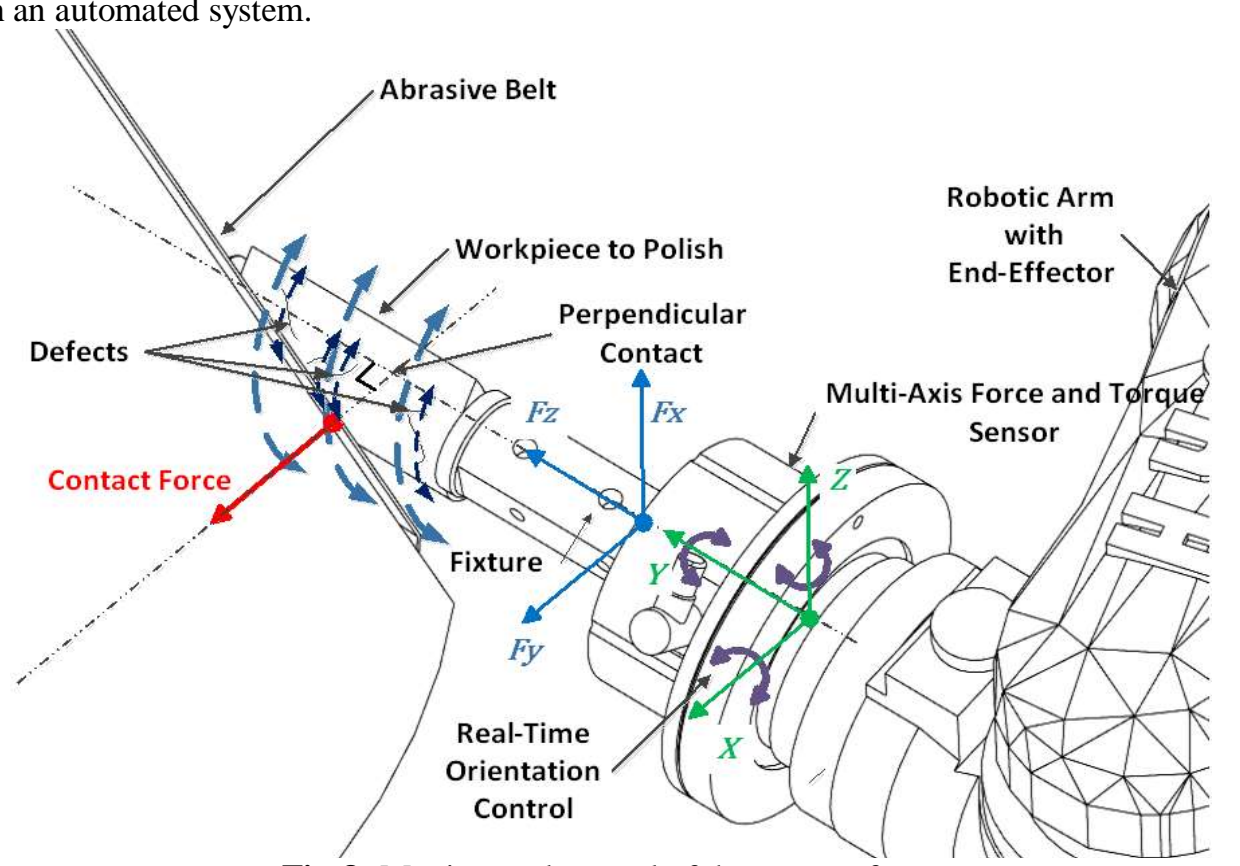

Fig-8: Monitor and control of the contact force 


\section{CONCLUSIONS}

The importance of polishing processes as part of main stream production lines in aerospace industry was discussed and the need for an alternative approach to the current manual processes have been explained. Lack of detailed understanding of manual polishing process was identified as a reason for failure in deploying existing automated polishing systems at industrial production environment. It was explained that the disconnect between existing automated polishing systems with the techniques and methods that manual operators use, has caused numerous limitations in automated systems.

An approach to capture polishing parameters and operational variables of manual polishing processes were proposed and discussed in this article. A set of laboratory experiments were carried out on small complex components, such as those used in aerospace industry. This was aimed at understanding what machining parameters are controlled by manual operators and when and under what circumstances the operators reconfigure the machining variable to obtain the operational quality required. The experiments' results were analyzed, interpreted and accordingly, a number of design considerations were proposed for development of an integrated robotic polishing system as part of this on-going research project [31].

It was also discussed that the current high quality manual polishing processes are highly dependent on the operators' skill level and may vary from one operator to another. In addition, the duration in which operators can maintain such a high-standard of skill level may significantly vary due to potentially hazardous operations, fatigues and human errors. Therefore, a significant potential was recognized in the application of robotic systems to automate some of the manual polishing operations. It is envisaged that such systems can bring a considerable consistency to the quality of polishing and can provide a flexible capacity that is essential for the current fluctuating market in this domain of industry. This is in spite of the fact that robots are currently much slower and significantly inflexible in comparison with skilled polishing operators.

\section{ACKNOWLEDGEMENT}

The authors acknowledge support from the EPSRC Centre for Innovative Manufacturing in Intelligent Automation, under grant reference number EP/IO33467/1.

\section{REFERENCES}

[1] A. Dickman, The science of scratches-Polishing and buffing mechanical surface preparation, Metal Finishing.

(2007). http://www.sciencedirect.com/science/article/pii/S0026 057607000487 (accessed February 5, 2016).

[2] Liang Liao, Fengfeng $\mathrm{Xi}$, A linearized model for control of automated polishing process, in: Proceedings of 2005 IEEE Conference on Control Applications, 2005 CCA 2005, IEEE, 2005: pp. 986-991. http://ieeexplore.ieee.org/lpdocs/epic03/wrapper.htm?a rnumber $=1507258$ (accessed February 5, 2016).

[3] A.R.A. Besari, A.S. Prabuwono, R. Zamri, M.D.M. Palil, Computer vision approach for robotic polishing application using artificial neural networks, in: 2010 IEEE Student Conference on Research and Development (SCOReD), IEEE, 2010: pp. 281-286. http://ieeexplore.ieee.org/lpdocs/epic03/wrapper.htm?a rnumber $=5704017$ (accessed February 5, 2016).

[4] H. Khakpour, L. Birglenl, Experimental Study on Abrasive Waterjet Polishing of Hydraulic Turbine Blades, IOP Conference Series: Earth and Environmental Science. http://iopscience.iop.org/article/10.1088/17551315/22/5/052016/meta (accessed February 26, 2016).

[5] Modern Machining Shop. Robotic Polishing of Turbine Engine Blades: Testing explores an airfoil finishing alternative to loose-abrasive polishing. (2015). http://www.mmsonline.com/articles/robotic-polishingof-turbine-engine-blades (accessed February 26, 2016).

[6] H. Executive, Safety, Safety in the use of abrasive wheels, HSE Books, 2000.

[7] H. Executive, Safety, Health and safety in engineering workshops, HSE Books, 1999.

[8] Wen-Jong Lin, Bryan Tsong-Jye Ng, Xiaoqi Chen, Zhiming Gong, Jingbing Zhang, KiewChoonMeng, Self-compensating closed loop real-time robotic polishing system, in: ICARCV 2004 8th Control, Automation, Robotics and Vision Conference, 2004, IEEE, 2004: pp. 199-204. doi:10.1109/ICARCV.2004.1468822.

[9] Kenton T. The future of mechanical surface finishing. Metal Finishing. 2009 May 31;107(5):22-4. (accessed February 5, 2016).

[10] D. a. Axinte, J. Kwong, M.C. Kong, Workpiece surface integrity of Ti-6-4 heat-resistant alloy when employing different polishing methods, Journal of Materials Processing Technology. 209 (2009) 1843-1852. doi:10.1016/j.jmatprotec.2008.04.046.

[11] P. Chotiprayanakul, D. Liu, G. Dissanayake, Humanrobot-environment interaction interface for robotic grit-blasting of complex steel bridges, Automation in Construction.

(2012). http://www.sciencedirect.com/science/article/pii/S0926 580512000702 (accessed December 28, 2015).

[12] X. Chen, Z. Gong, H. Huang, Development of robotic system for 3D profile grinding and polishing, SIMTech, Singapore, Tech Rep AT/00/012/AMP. (2000). (accessed February 5, 2016).

[13] H. Huang, Z.. Gong, X.. Chen, L. Zhou, Robotic grinding and polishing for turbine-vane overhaul, Journal of Materials Processing Technology. 127 (2002) 140-145. doi:10.1016/S0924-0136(02)00114-0.

[14] A. Guiot, S. Pattofatto, C. Tournier, L. Mathieu, Modeling of a Polishing Tool to Simulate Material Removal, Advanced Materials Research. 223 (2011) 754-763. http://www.scientific.net/AMR.223.754

[15] D.A. Davidson, Mass finishing processes, Metal Finishing. $\quad 99 \quad$ (2001) 110-124 http://www.sciencedirect.com/science/article/pii/S0026 057601852685 (accessed March 2, 2016). 
[16] L. Gillespie, Mass Finishing Handbook, Industrial Press, 2006. (accessed March 2, 2016).

[17] M. Murphy, Technical developments in 1999: Inorganic "metallic" finishes, processes, and equipment, Metal Finishing. (2000). http://www.sciencedirect.com/science/article/pii/S0026 057600813896 (accessed January 12, 2016).

[18] M.J. Tsai, J.-L. Chang, J.-F. Haung, Development of an Automatic Mold Polishing System, IEEE Transactions on Automation Science and Engineering. 2 (2005) 393-397.

http://ieeexplore.ieee.org/lpdocs/epic03/wrapper.htm?a rnumber=1514458 (accessed February 5, 2016).

[19] X. Chen, Z. Gong, H. Huang, L. Zhou, An automated 3D polishing robotic system for repairing turbine airfoils, Industrial Automation Journal. (1999). http://citeseerx.ist.psu.edu/viewdoc/download?doi=10.

1.1.196.1656\&rep=rep1\&type=pdf (accessed February $9,2016)$.

[20] X. Chen, D. Wang, S. Bai, W. Lin, Hybrid linear compensation and improved Hermite interpolation for optical measurement of 3D airfoil surface, Robotics, Automation and Mechatronics, 2004 IEEE Conference. (2004). pp. 197-201 vol.1. doi: 10.1109/RAMECH.2004.1438916

http://ieeexplore.ieee.org/xpls/abs_all.jsp?arnumber=14 38916 (accessed February 9, 2016).

[21] B. Ng, W. Lin, X. Chen, Intelligent system for turbine blade overhaul using robust profile re-construction algorithm. Control, Automation, Robotics and Vision Conference, 2004. ICARCV 2004 8th, 2004, pp. 178183 Vol. 1. doi: 10.1109/ICARCV.2004.1468819 http://ieeexplore.ieee.org/xpls/abs_all.jsp?arnumber=14 68819 (accessed February 9, 2016).

[22] H. Huang, Z.M. Gong, X.Q. Chen, L. Zhou, SMART Robotic System for 3D Profile Turbine Vane Airfoil Repair Process, International Journal of Advanced Manufacturing Technology. 21 (2003) 275-283. http://link.springer.com/article/10.1007/s00170030003 2.

[23] H. Du, Y. Sun, D. Feng, J. Xu, Automatic robotic polishing on titanium alloy parts with compliant force/position control, Proceedings of the Institution of Mechanical Engineers, Part B: Journal of Engineering Manufacture. $229 \quad$ (2015) 1180-1192. doi: $10.1177 / 0954405414567518$.

[24] Fraunhofer, poliMATIC: AUTOMATED POLISHING FOR THE EUROPEAN TOOL INDUSTRY, (2013). http://www.automated-polishing.eu/.

[25] Archos, ARCOS System for Surface Finishing. http://www.arcossrl.com/_modules/download/downloa d/brochure/broch_eng.pdf.

[26] SHL Automatisierungstechnik AG, Core expertise the perfect surface: automated polishing. (2015). http://www.shlautomatisierung.de/cms/us/automated_polishing/

[27] RJH Finishing System. Ltd., Finishing Machines: Semi-Automatic Rise \& Fall Grinder. Wallaby - WAL Series. $\quad$ http://www.rjhfinishing.co.uk/finishingmachines/machines/power-grinders/wallaby.

[28] Vulcan Engineering CO., VTSTM - VULCAN TACTILE SYSTEM. 2/robotics/metal-removal-robots/vts-vulcan-tactilesystem/

[29] Usman, Z., Monfared, R.P., Lohse N., \& Jackson, M., (2016). "An investigation of highly accurate and precise robotic hole measurements using non-contact devices." Int. J. Metrology and Quality Engineering $7(2)$.

[30] Ilangovan, B., Monfared, R.P. \& Jackson, M., “An automated solution for fixtureless sheet metal forming”, Int J AdvManufTechnol (2016) 82: 315. doi:10.1007/s00170-015-7366-X

[31] Eugene B.F. Kalt, Radmehr P. Monfared, Michael R. Jackson, Development of an intelligent automated polishing system, euspen's 16th International Conference \& Exhibition, Nottingham, UK, 2016

http://www.vulcangroup.com/products- 